\title{
Présence de champignons du genre Coelomomyces chez des Anopheles en Haute-Volta
}

\author{
( $3^{e}$ note $)$
}

\author{
par F. RODHAIN et J. BRENGUES
}

Unité d'Ecologie virale. Institut Pasteur, 25, rue du $D^{r}-R o u x, F 75015$ Paris

et Entomologie médicale, O.R.S.T.O.M., 70-74, route d'Aulnay, F 93140 Bondy

\section{Résumé}

Les auteurs rapportent la présence, en Haute-Volta, de quatre espèces de champignons appartenant au genre Coelomomyces, et parasitant des femelles d'Anopheles gambiae: C. africanus, $C$. grassei, $C$. ascariformis et $C$. walkeri (?). Tous ces parasites étaient déjà connus, chez le même hôte, d'autres localités en Afrique subsaharienne.

\section{Summary}

Presence in Anopheles from Haute-Volta (Africa) of fungus from the genus Coelomomyces $\left(3^{\mathrm{e}}\right.$ note $)$.

The authors record the presence, in Upper Volta, of four species of Coelomomyces fungus, parasitic in females A. gambiae: C. africanus, C. grassei, C. ascariformis et C. walkeri (?). All these parasites were known, in the same host, from other places in Africa south of the Sahara.

Faisant suite à deux publications antérieures rapportant la présence de Coelomomyces (Blastocladiales: Coelomomycetaceae) chez des larves d'Anopheles (Rodhain, 1969 ; Rodhain et Gayral, 1971) en Haute-Volta, la présente note fait état de parasitisme d'Anopheles adultes femelles par ce même genre de champignons. 
C'est au cours de dissection de culicidés, dans le cadre d'une étude sur la filariose lymphatique que l'un de nous (J. Brengues) put observer la présence du champignon au niveau des ovaires de səpt femelles d'Anopheles gambiae Giles. Quatre espèces de Coelomomyces ont ainsi été trouvées.

\section{Cas I et II -}

Hôtes : A. gambiae femelles, rézoltées l'une (nº HV. 1508) à Kampiti (cercle de Dori) le 17 août 1968, l'autre ( ${ }^{\circ}$ HV. 1509) à Torodi (cercle de Dori) le 24 octobre 1968 (gîte larvaire probable : trou à «banco »).

Parasite : Localisé au niveau des ovaires des insectes. Pas de mycélium visible. Sporanges tous semblables, ovalaires, asymétriques: légèrement aplatis sur une face, mesurant dans un cas 34,1 $\mu$ à 40,3 $\mu$ de long, 21,7 $\mu$ à 24,8 $\mu$ de large, dans l'autre cas : $24,8 \mu$ à $31 \mu$ de long, $12,4 \mu$ à 18,6 $\mu$ de large. Malgré un assez mauvais état de conservation, l'étude de ces sporanges révèle l'existence de faibles cannelures anastomosées. Une fente de déhiscence longitudinale est souvent visible.

$\mathrm{Au}$ total, l'aspect est comparable à celui de deux cas que nous avons déjà eu l'occasion de rapporter en Haute-Volta chez des larves d'Anopheles rufipes Gough et d'Anopheles squamosus Theobald et pour lesquels l'identification proposée était celle de Coelomomyces africanus. Rappelons que celle dénomination a été proposée à l'origine pour le type 3 décrit chez A. gambiae en Sierra Leone par Walker (1938) ; ce parasite fut ensuite retrouvé à plusieurs reprises dans différents pays d'Afrique, toujours chez des Anophéles: Anopheles funestus Giles et A. gambiae au Kenya (Haddow, 1942), A. squamosus en Angola et peut-être A. gambiae en Ouganda (Gibbins, 1932); Couch et Umphlett (1963) citent encore: $A$. funestus au Liberia (Gilioli et al., 1956 ; Darwish et al., 1960), A. squamosus à Madagascar (Grjebine et al., 1953).
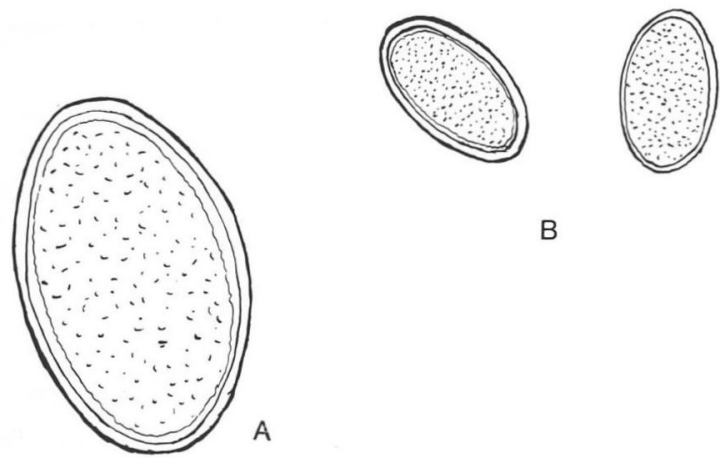

B

Fig. 1. - A : Coelomomyces walkeri (?) : sporange mesurant 58,9 u sur 40,3 $\mu$ (HV. 1514). B : Coelomomyces africanus: sporanges; longueur: $29,4 \mu$ et $26,3 \mu$ (HV. 1508) 


\section{Cas III et IV -}

Hôtes : deux A. gambiae femelles (n ${ }^{\circ}$ HV. 1510 et HV. 1511) capturées le 28 août 1970 à Tingréla (cercle de Banfora).

Parasite : localisé aux ovaires. Mycélium présent en faible quantité chez l'un des anophèles, non visible chez l'autre. Deux types de sporanges :

- sporanges à paroi épaisse, ovalaires, de couleur brune, à surface finement ponctuée ; surface ornée de huit côtes longitudinales, de section semi-circulaire, dont cinq s'anastomosent à un pôle ; taille : 43,4 $\mu$ à $70 \mu$ de long, $31 \mu$ à 45,6 $\mu$ de large ; fente de déhiscence longitudinale, entre deux côtes ;

- sporanges à paroi lisse, ovalaires, symétriques, de couleur plus claire, de taille variable : $37,2 \mu$ à $43,4 \mu$ de long et $24,8 \mu$ à $27,9 \mu$ de large pour l'un, $50 \mu$ à $74,2 \mu$ de long et $37,2 \mu$ à $49,3 \mu$ de large pour l'autre.

Au total l'aspect de ce parasite, et surtout celui des sporanges a paroi épaisse évoque immédiatement un Coelomomyces déjà décrit par Rioux et Pech (1960) du Tchad, chez $A$. gambiae également : $C$. grassei. 11 s'agit, à notre connaissance, de la première mention de ce parasite ailleurs que dans les localités d'où il fut décrit pour la première fois.

\section{Cas V -}

Hôte : A. gambiae femelle ( ${ }^{\circ}$ HV. 1512) de Pala (cercle de Bobo-Dioulasso), le 19 août 1967.

Parasite : localisé également aux ovaires de l'insecte. Pas de mycélium visible. Deux types de sporanges:

- sporanges ovalaires, d'un brun clair, présentant une ornementation constituée par un reticulum délimitant des aires polygonales juxtaposées; paroi apparaissant, de profil, très légèrement festonnée; fente de déhiscence longitudinale ; taille : $40,3 \mu$ à $55,8 \mu$ de long, $24,8 \mu$ à $31 \mu$ de large ;

- sporanges à paroi lisse, moins nombreux, de même couleur, à contenu granuleux ; taille : $46,5 \mu$ à $62 \mu$ de long, $27,9 \mu$ à $34,1 \mu$ de large.

Ces sporanges rappellent, en plus petit, ceux que nous avons déjà observés chez des larves d'A. gambiae de la même localité (n ${ }^{\circ}$ HV. 1506 et HV. 1507 : Rodhain et Gayral, 1971). Ils présentent un aspect tout à fait comparable à celui figuré dans la publication de Walker (type 4, pl. IV), et qui fut appelé C. ascariformis par Van Thiel (1954); cet auteur regroupait sous cette dénomination le type 4 de Walker et un parasite décrit des Philippines par Manalang en 1930. On sait que, par la suite, Laird (1959) rapporta le parasite de Manalang à $C$. indiana Iyengar. 
244

F. RODHAIN ET J. BRENGUES
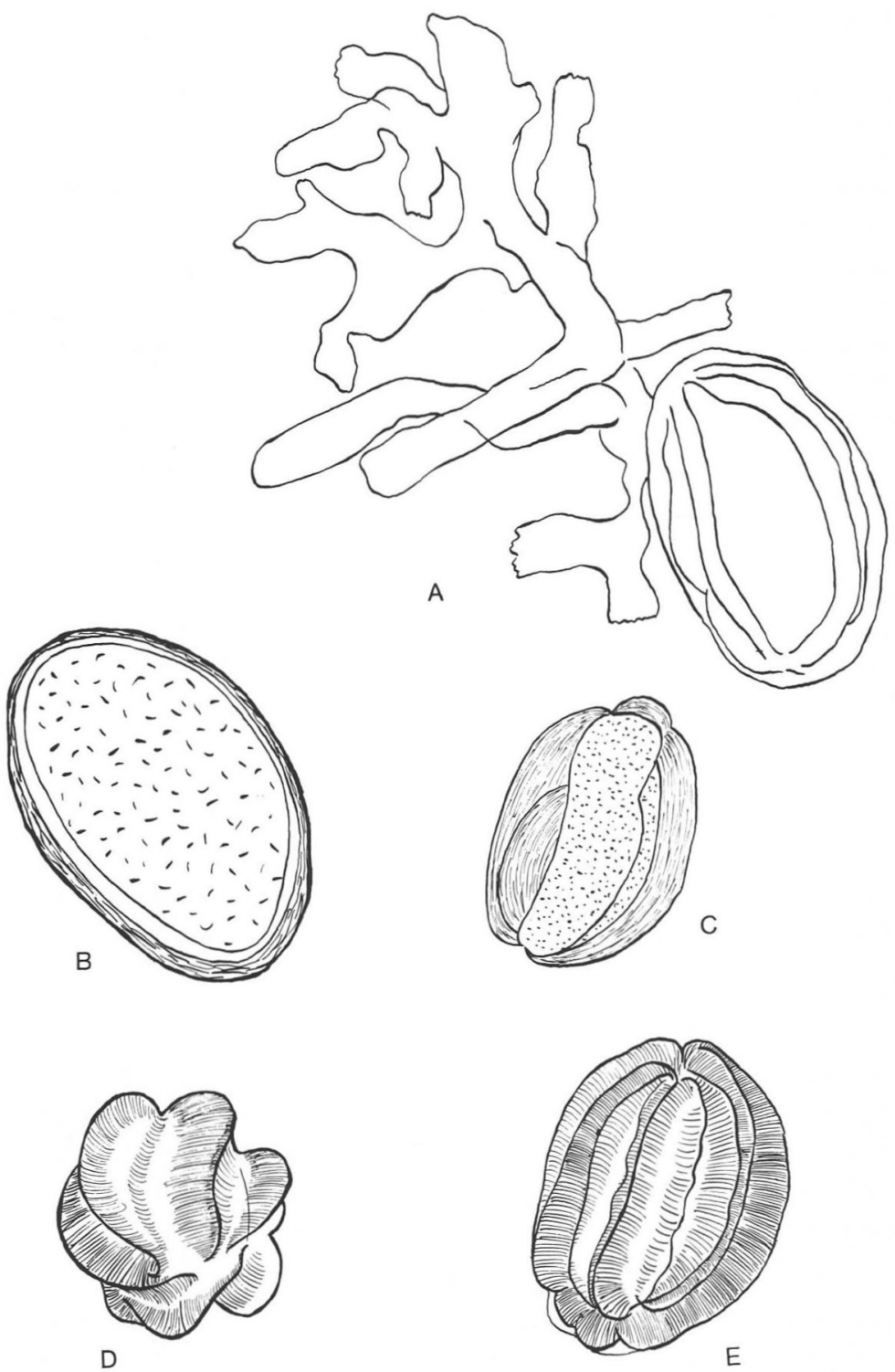

FIG. 2. - Coelomomyces grassei. A : fragment de mycelium et sporange attenant (HV. 1511). B : Sporange à paroi lisse, long.: $74,2 \mu$ (HV. 1511). C : sporange à paroi épaisse, long. : $55,8 \mu$ (HV. 1511). D. : sporange à paroi épaisse en vue subpolaire (HV. 1510. E. : sporange à paroi épaisse, long. : $62 \mu$ (HV. 1510)

Les dessins des figures 1 et 2 ont été effectués à la même échelle. 


\section{Cas VI et VII -}

Hôtes : A. gambiae femelles, l'une (n ${ }^{\circ}$ HV. 1513) de Pala (cercle de BoboDioulasso), le 29 septembre 1967, l'autre ( ${ }^{\circ}$ HV. 1514) de Tingréla (cercle de Banfora), le 23 août 1967.

Parasite : localisé aux ovaires du moustique. Pas de mycélium visible. Sporanges ovalaires, d'un brun pâle, à paroi lisse sans cannelure ni ornementation visible, mesurant dans un cas de $58 \mu$ à $65 \mu$ de long sur $37,2 \mu$ à $46,5 \mu$ de large, dans l'autre cas de $43,4 \mu$ à $62 \mu$ de long sur $31 \mu$ à $37,2 \mu$ de large.

Ces sporanges font évoquer deux parasites africains : le «type c» de Muspratt (1946) et le type 1 de Walker (1938). En raison de leur taille, nous pensons pouvoir les rapprocher de cette dernière espèce qui fut identifiée à Coelomomyces walkeri Van Thiel 1954.

En conclusion, nous sommes en présence de quatre espèces de Coelomomyces parasitant les femelles d'A. gambiae en Haute-Volta :

- C. africanus, parasite largement répandu en Afrique subsaharienne. et qui semble spécifique des Anopheles;

- C. grassei, qui n'était connu jusqu'ici que du Tchad, chez des larves d'A. gambiae ;

- C. ascariformis, un champignon déjà observé chez $A$. gambiae en Sierra Leone et en Haute-Volta;

- C. walkeri (probablement), lui aussi connu de Sierra Leone chez le même hôte.

\section{Bibliographie}

Couch (J. N.) and Umphlett (C. J.), 1963. - Coelomomyces infections. In : Steinhaus, E. A. : Insect Pathology, an advanced treatise. Academic Press publ., New York and London, 2, 149-188.

Gibbins (E. G.), 1932. - Natural malaria infection of house frequenting Anopheles mosquitoes in Uganda. Ann. Trop. Med. Parasit., 26, 239-266.

HadDow (A. J.), 1942. - The mosquito fauna and climate of native huts at Kisumu, Kenya. Bull. Ent. Res., 33, 91-142.

LAIRD (M.), 1959. - Fungal parasites of mosquito larvae from the Oriental and Australian regions, with a key to the genus Coelomomyces (Blastocladiales: Coelomomycetaceae). Canad. J. Zool., 37, 781-791.

Manalang (G.), 1930. - Coccidiosis in anopheline mosquitoes. Philipp. J. Sci., 42, 279-282.

Muspratt (J.), 1946. - On Coelomomyces fungi causing high mortality of Anopheles gambiae larvae in Rhodesia. Ann. Trop. Med. Parasit., 40, 10-17.

Annales de Parasitologie humaine et comparée (Paris), t. 49, $\mathrm{n}^{\circ} 2$ 
Rioux (J.-A.) et PeCH (J.), 1960. - Coelomomyces grassei n. sp. parasite d'Anopheles gambiae Giles (note préliminaire). Acta Trop., 17, 179-182.

Rodhain (F.) et Gayral (P.), 1971. - Sur la présence d'un champignon du genre Colomomyces en République de Haute-Volta. Ann. Parasit. hum. comp., 44, 261-264.

Rodhain (F.), et Gayral (P.), 1971. - Nouveaux cas de parasitisme de larves d'Anopheles par des champignons du genre Coelomomyces en République de Haute-Volta. Ann. Parasit. hum. comp., 46, 295-300.

VAN Thiel (P. H.), 1954. - Trematode, Gregarine and fungus parasites of Anopheles mosquitoes. J. Parasit., 40, 271-279.

WALKer (A. J.), 1938. - Fungal infections of mosquitoes, especially of Anopheles costalis. Ann. Trop. Med. Parasit., 32, 231-244. 